\title{
TALES ABOUT TAILS ${ }^{1}$
}

\author{
HAAKON WAADELAND
}

\begin{abstract}
Necessary and sufficient conditions are given for a sequence $\left\{g^{(n)}\right\}$ to be a sequence of tails of a convergent continued fraction. Some special cases are also studied.
\end{abstract}

0. Introduction. The problems to be discussed in the present paper all have to do with continued fractions

$$
\underset{n=1}{\infty} \frac{a_{n}}{1}, \quad a_{n} \in \mathbf{C}, a_{n} \neq 0, n=1,2,3, \ldots
$$

For any fixed $n$ the extended complex number

$$
f_{n}=\stackrel{K}{K=1}_{\nu}^{n} \frac{a_{\nu}}{1}, \quad f_{0}=0,
$$

is called the $n$th approximant of the continued fraction $(0.1)$. In case of convergence the limit

$$
\lim _{n \rightarrow \infty} f_{n}=f
$$

exists and is called the value of the continued fraction $(0.1)$. The word "approximant" is meaningful, and the sequence $\left\{f_{n}\right\}_{n=1}^{\infty}$ of approximants exists regardless of whether or not $(0.1)$ converges.

If, in particular, the continued fraction $(0.1)$, and hence all its tails,

$$
\stackrel{\infty}{K} \underset{\nu=n+1}{a_{\nu}},
$$

converge (possibly to $\infty$ ), then the sequence $\left\{f^{(n)}\right\}_{n=0}^{\infty}$ of extended complex numbers, where $f^{(n)}$ is the value of $(0.4), f^{(0)}=f$, is called the sequence of right tails of the convergent continued fraction $(0.4)$. Obviously the numbers $f^{(n)}$ satisfy the set of recursion relations

$$
f^{(n)}=\frac{a_{n+1}}{1+f^{(n+1)}}, \quad n=0,1,2, \ldots
$$

understood such that in the particular case $f^{(n)}=\infty$, we have $f^{(n+1)}=-1$. For a given convergent continued fraction ( 0.1$)$ (convergence to $\infty$ being permitted) the

Received by the editors January 4, 1983.

1980 Mathematics Subject Classification. Primary 30B70; Secondary 40A15.

Key words and phrases. Continued fraction, right tails, wrong tails.

'Supported by the Norwegian Research Council for Science and the Humanities.

$$
\begin{aligned}
& \text { c1984 American Mathematical Society } \\
& 0002-9939 / 84 \$ 1.00+\$ .25 \text { per page }
\end{aligned}
$$


sequence of right tails is unique. The condition $a_{n} \neq 0, n=1,2,3, \ldots$, which is part of the definition, is essential. Any other sequence of extended complex numbers satisfying $(0.5)$, i.e. any sequence $\left\{g^{(n)}\right\}_{n=0}^{\infty}$ with $g^{(0)} \neq f^{(0)}$ and such that

$$
g^{(n)}=\frac{a_{n+1}}{1+g^{(n+1)}} \quad \text { for all } n \geqslant 0
$$

is called a sequence of wrong tails. Tails of continued fractions (also wrong tails) are of vital importance for modifications of continued franctions. The survey article [13] discusses modifications and their applications, and also contains an extensive bibliography. Newer results are found in [4-7].

In [10, Theorem 2.7] the following question is discussed: Which sequences $\left\{f_{n}\right\}_{n=1}^{\infty}$ are sequences of approximants for some continued fraction $b_{0}+K_{n=1}^{\infty} a_{n} / b_{n}$ ? The answer is that "almost any sequence is". The only conditions that have to be satisfied are $f_{0} \neq \infty$ and $f_{n-1} \neq f_{n}$ for all $n \geqslant 1$. For continued fractions $b_{0}+K\left(a_{n} / 1\right)$ the conditions are $f_{0} \neq \infty$ and $f_{n-1} \neq f_{n}, f_{n-1} \neq f_{n+1}$ for all $n \geqslant 1$.

In the present paper we shall raise the corresponding question for tails:

Which sequences $\left\{g^{(n)}\right\}_{n=0}^{\infty}$ are sequences of right tails for some (convergent) continued fraction?

In view of $\left(0.5^{\prime}\right)$ we have

$$
g^{(n)}\left(1+g^{(n+1)}\right)=a_{n+1} \text { for all } n \geqslant 0
$$

Since all $a_{n} \neq 0$, we have $\left(g^{(n)}=0\right) \Leftrightarrow\left(g^{(n+1)}=\infty\right)$ and $\left(g^{(n)}=\infty\right) \Leftrightarrow\left(g^{(n+1)}=\right.$ $-1)$, but in neither of these cases $a_{n+1}$ is uniquely determined. In the present paper we shall avoid this situation by assuming that $g^{(n)} \neq 0, \infty$ for all $n \geqslant 0$, and $g^{(n)} \neq-1$ for all $n \geqslant 1$, in which case the continued fraction is uniquely determined as

$$
\frac{g^{(0)}\left(1+g^{(1)}\right)}{1}+\frac{g^{(1)}\left(1+g^{(2)}\right)}{1}+\frac{g^{(2)}\left(1+g^{(3)}\right)}{1}+\cdots
$$

The sequence $\left\{g^{(n)}\right\}$ is a sequence of right tails for this if and only if (0.7) converges to $g^{(0)}$. This is how the problem will be attacked in the next section.

Why is this problem interesting? The method of acceleration of convergence, as presented in [4 and 5], and further extended in [6], uses auxiliary (convergent) continued fractions, where we need to know the value of all tails. The method thus heavily depends upon the knowledge of such auxiliary continued fractions. If the supply of known continued fractions with known tails increases, then the possibility of using the method also increases. A complete, or even a partial answer to the question raised in the present paper obviously makes it possible to construct continued fractions with known tails. (A different type of investigation of tails is presented in [8].) 
1. The main result. It turns out that under the additional conditions mentioned above the question can easily be settled completely.

THEOREM 1. Let $\left\{g^{(n)}\right\}_{n=0}^{\infty}$ be a sequence of complex numbers, all $\neq 0$ and all except possibly $\mathrm{g}^{(0)}$ different from - 1. Furthermore, let

$$
\kappa_{n}=-\frac{1+g^{(n)}}{g^{(n)}} \text { for } n=1,2,3, \ldots
$$

Then $\left\{g^{(n)}\right\}_{n=0}^{\infty}$ is the sequence of right tails for some continued fraction if and only if

$$
1+\kappa_{1}+\kappa_{1} \kappa_{2}+\kappa_{1} \kappa_{2} \kappa_{3}+\cdots=\infty .
$$

(This theorem is related to [11, Satz 2.45].)

Proof. From the previous section we know that $\left\{g^{(n)}\right\}$ is the sequence of right tails for some continued fraction (which has to be $(0.7)$ ) if and only if $(0.7)$ converges to $g^{(0)}$. Let in $(0.1)$

$$
a_{n+1}=g^{(n)}\left(1+g^{(n+1)}\right), \quad n \geqslant 0,
$$

and let $A_{n}$ and $B_{n}$ have the standard meaning, given by

$$
f_{n}=\frac{A_{n}}{B_{n}}, \quad n \geqslant 0
$$

and the initial conditions

$$
A_{0}=0, \quad A_{1}=a_{1}, \quad B_{0}=1, \quad B_{1}=1 .
$$

From [9, Propositions 1 and 2] we know that

$$
A_{n}-B_{n} g^{(0)}=(-1)^{n-1} \prod_{m=0}^{n} g^{(m)}
$$

and

$$
B_{n}=\sum_{m=1}^{n+1}(-1)^{n+1-m}\left(\prod_{\nu=m}^{n} g^{(\nu)}\right)\left(\prod_{\nu=1}^{m-1}\left(1+g^{(\nu)}\right)\right)
$$

(see also [15, p. 47]). If we divide (1.6) by (1.7) and use (1.4) and (1.1) we get

$$
f_{n}-g^{(0)}=\frac{-g^{(0)}}{1+\kappa_{1}+\kappa_{1} \kappa_{2}+\cdots+\kappa_{1} \kappa_{2} \cdots \kappa_{n}},
$$

from which the theorem follows immediately.

REMARK. We may actually squeeze much more information out of (1.8). If we briefly call the series $1+\kappa_{1}+\kappa_{1} \kappa_{2}+\cdots$ "the $\kappa$ series", the sequence $\left\{g^{(n)}\right\}$ merely 
"the $g$-sequence" and let "the continued fraction" mean (0.7), we get some results of the type:

$$
\begin{aligned}
& \text { Property of } g \text {-sequence } \\
& \text { Property of } \kappa \text {-series } \\
& { }^{\searrow} \text { Property of continued fraction. }
\end{aligned}
$$

\begin{tabular}{|c|c|c|}
\hline$\kappa$-series & $g$-sequence & Continued fraction \\
\hline converges to $\infty$ & $\begin{array}{l}\text { is a sequence of right } \\
\text { tails of a continued } \\
\text { fraction }\end{array}$ & converges to $g^{(0)}$ \\
\hline converges to 0 & $\begin{array}{l}\text { is a sequence of wrong } \\
\text { tails of a continued } \\
\text { fraction }\end{array}$ & converges to $\infty$ \\
\hline $\begin{array}{l}\text { converges to } \\
H_{0} \neq 0, \infty\end{array}$ & $\begin{array}{l}\text { is a sequence of wrong } \\
\text { tails of a continued } \\
\text { fraction }\end{array}$ & $\begin{array}{l}\text { converges to } \\
g^{(0)}\left(1-1 / H_{0}\right)\end{array}$ \\
\hline diverges & $\begin{array}{l}\text { is a sequence of wrong } \\
\text { tails of a continued } \\
\text { fraction }\end{array}$ & diverges \\
\hline
\end{tabular}

We list them in the following way:

2. A result on wrong tails. From the remark at the end of the previous section we find that if the $\kappa$-series converges to an $H_{0} \neq \infty$, then the continued fraction converges (possibly to $\infty$ ). Being a convergent continued fraction it has a unique sequence of right tails $f^{(n)}$. If the $\kappa$-sequence converges to $H_{0} \neq \infty$ we have

$$
f^{(0)}=g^{(0)}\left(1-1 / H_{0}\right) .
$$

Since all $\kappa_{n} \neq 0$, it follows from the convergence of

$$
1+\kappa_{1}+\kappa_{1} \kappa_{2}+\kappa_{1} \kappa_{2} \kappa_{3}+\cdots
$$

to an $N_{0} \neq \infty$ that all the series

$$
1+\kappa_{n+1}+\kappa_{n+1} \kappa_{n+2}+\kappa_{n+1} \kappa_{n+2} \kappa_{n+3}+\cdots
$$

converge to numbers $H_{n} \neq \infty$, and it follows trivially that

$$
f^{(n)}=g^{(n)}\left(1-1 / H_{n}\right), \quad n=0,1,2, \ldots
$$

(Observe that (2.3) also holds if $H_{0}=\infty$, in which case all $H_{n}=\infty$. (2.3) then reduces to $f^{(n)}=g^{(n)}$.)

We thus have a procedure for computing the right tails of a convergent continued fraction $(0.1)$ if we know an arbitrary sequence $\left\{g^{(n)}\right\}_{n=0}^{\infty}$ of wrong tails with $g^{(n)} \neq 0,-1, \infty$. Obviously we cannot expect $f^{(n)}$ to be different from $0,-1, \infty$. 
On this background the next theorem, giving sufficient conditions for a sequence to be a sequence of wrong tails for a convergent continued fraction, is of a certain interest.

THEOREM 2. Let $r$ be an arbitrary positive number $<1$, and let $D_{r}$ be the disk

$$
D_{r}=\left\{w\left|w+\frac{1}{1-r^{2}}\right| \leqslant \frac{r}{1-r^{2}}\right\} .
$$

Let $\left\{g^{(n)}\right\}_{n=0}^{\infty}$ be a sequence of complex numbers, all located in $D_{r}$. Then $\left\{g^{(n)}\right\}_{n=0}^{\infty}$ is a sequence of wrong tails of a convergent continued fraction.

Proof. Simple computation shows that

$$
g^{(n)} \in D_{R} \Leftrightarrow\left|\kappa_{n}\right| \leqslant r .
$$

Hence if $g^{(n)} \in D_{r}$, the series

$$
1+\kappa_{1}+\kappa_{1} \kappa_{2}+\kappa_{1} \kappa_{2} \kappa_{3}+\cdots
$$

converges to a number $\leqslant 1 /(1-r)<\infty$, and the statement in the theorem follows from the remarks after Theorem 1.

Theorem 2 is a convergence theorem for continued fractions $(0.1)$, where sufficient conditions for convergence are given in terms of numbers $g^{(n)}$, such that $a_{n+1}=$ $g^{(n)}\left(1+g^{(n+1)}\right)$. Related theorems are proved by Scott and Wall, Paydon and Wall, Pringsheim, Perron and Van Vleck (see [15, Theorem 11.1-4]). Also, in these theorems an essential role is played by the sum $\left(2.2^{\prime}\right)$ (in a more special case).

3. Examples. In the present section we shall study three special cases in connection with Theorem 1.

(a) Periodic sequence. Assume in addition to the conditions of Theorem 1 that the sequence $\left\{g^{(n)}\right\}_{n=0}^{\infty}$ is periodic with period $k$. By studying the partial sums $S_{n k+p}$, $p=0,1,2, \ldots, k-1$ of the $\kappa$-series it follows immediately from Theorem 1 that the $g$-sequence is a sequence of right tails of a convergent continued fraction if and only if

$$
\left\{\begin{array}{c}
\kappa_{1} \cdot \kappa_{2} \cdots \kappa_{k}=1 \\
\text { or } \\
\left|\kappa_{1} \cdot \kappa_{2} \cdots \kappa_{k}\right|>1
\end{array}\right\} \text { and }\left\{\begin{array}{c}
1+\kappa_{1}+\kappa_{1} \kappa_{2}+\cdots+\kappa_{1} \cdots \kappa_{k-1} \neq 0 \\
1+\kappa_{2}+\kappa_{2} \kappa_{3}+\cdots+\kappa_{2} \cdots \kappa_{k} \neq 0 \\
1+\kappa_{k}+\kappa_{k} \kappa_{1}+\cdots+\kappa_{k} \kappa_{1} \cdots \kappa_{k-2} \neq 0
\end{array}\right\}
$$

(For $k=1$ or $k=2$ the condition to the right is empty.) Before leaving the periodic case we shall look at the transition from wrong tails to right tails in this case. For simplicity we shall restrict ourselves to the case $k=3$. But even the general case does not offer any problem. We assume, according to the remark after Theorem 1, that the $\kappa$-series converges to an $H_{0} \neq \infty$. In addition, we shall assume that the continued fraction converges to a finite number, in which case we also know that $H_{0} \neq 0$. The $\kappa$-series can be rearranged in the following way

$$
\left(1+\kappa_{1}+\kappa_{1} \kappa_{2}\right)\left(1+\kappa_{1} \kappa_{2} \kappa_{3}+\left(\kappa_{1} \kappa_{2} \kappa_{3}\right)^{2}+\cdots\right) .
$$


Since this converges to a finite number $H_{0} \neq 0$ we have

$$
1+\kappa_{1}+\kappa_{1} \kappa_{2} \neq 0 \text { and }\left|\kappa_{1} \kappa_{2} \kappa_{3}\right|<1 \text {, }
$$

and hence

$$
H_{0}=\frac{1+\kappa_{1}+\kappa_{1} \kappa_{2}}{1-\kappa_{1} \kappa_{2} \kappa_{3}} \text {. }
$$

Similarly we get

$$
H_{1}=\frac{1+\kappa_{2}+\kappa_{2} \kappa_{3}}{1-\kappa_{1} \kappa_{2} \kappa_{3}}, \quad H_{2}=\frac{1+\kappa_{3}+\kappa_{3} \kappa_{1}}{1-\kappa_{1} \kappa_{2} \kappa_{3}} .
$$

( $H_{1}$ or $H_{2}$ may possibly be 0 .) From (2.3) we find

$$
\left\{\begin{array}{l}
f^{(0)}=g^{(0)} \frac{\kappa_{1}\left(1+\kappa_{2}+\kappa_{2} \kappa_{3}\right)}{1+\kappa_{1}+\kappa_{1} \kappa_{2}}, \\
f^{(1)}=g^{(1)} \frac{\kappa_{2}\left(1+\kappa_{3}+\kappa_{3} \kappa_{1}\right)}{1+\kappa_{2}+\kappa_{2} \kappa_{3}}, \\
f^{(2)}=g^{(2)} \frac{\kappa_{3}\left(1+\kappa_{1}+\kappa_{1} \kappa_{2}\right)}{1+\kappa_{1}+\kappa_{3} \kappa_{1}} .
\end{array}\right.
$$

We find similar formulas in the general $k$-case. For $k=1$ the formulas reduce to the well-known $f^{(0)}=-\left(1+g^{(0)}\right)$, and for $k=2$ they are $f^{(0)}=-\left(1+g^{(1)}\right), f^{(1)}=$ $-\left(1+g^{(0)}\right)($ see $[13$, p. 64]).

(b) Positive sequences. Assume in addition to the conditions on Theorem 1 that all $g^{(n)}$ are positive. Let $S_{2 m}$ and $S_{2 m+1}$ denote partial sums of the $\kappa$-series. Then

$$
\begin{aligned}
-S_{2 m} & =-\left(1+\kappa_{1}+\kappa_{1} \kappa_{2}+\kappa_{1} \kappa_{2} \kappa_{3}+\cdots+\kappa_{1} \kappa_{2} \cdots \kappa_{2 m-1}\right) \\
& =\frac{1}{g^{(1)}}+\frac{\kappa_{1} \kappa_{2}}{g^{(3)}}+\cdots+\frac{\kappa_{1} \kappa_{2} \cdots \kappa_{2 m-2}}{g^{(2 m-1)}}>\sum_{k=1}^{m} \frac{1}{g^{(2 k-1)}} .
\end{aligned}
$$

Similarly, we get $S_{2 m+1}>1+\sum_{k=1}^{m} 1 / g^{(2 k)}$. Hence

THEOREM 3. If $\left\{g^{(n)}\right\}_{n=0}^{\infty}$ is a sequence of positive numbers such that

$$
\sum_{m=1}^{\infty} \frac{1}{g^{(2 m)}}=\infty \text { and } \sum_{m=1}^{\infty} \frac{1}{g^{(2 m+1)}}=\infty,
$$

Then $\left\{g^{(n)}\right\}_{n=0}^{\infty}$ is the sequence of right tails for the continued fraction (0.7).

COROLlaRY 4. If $\left\{g^{(n)}\right\}_{n=0}^{\infty}$ is a monotone sequence of positive numbers such that

$$
\sum_{n=1}^{\infty} \frac{1}{g^{(n)}}=\infty,
$$

then $\left\{g^{(n)}\right\}_{n=0}^{\infty}$ is the sequence of right tails for the continued fraction (0.7).

An example to Corollary 4 is given by

$$
g^{(n)}=\alpha n+\beta, \quad \alpha>0, \beta>0 .
$$


It follows that the continued fraction

$$
K_{n=0}^{\infty} \frac{(\alpha n+\beta)(\alpha n+\alpha+\beta+1)}{1}
$$

converges to $\beta$, regardless of $\alpha$. This is likely to be known, but is hard to find in the literature. It does not seem to be contained in for instance [11, Chapter VI], where one might expect to find it.

(c)

$$
g^{(n)}=-\frac{1}{2}-\frac{L}{4(1+n)}, \quad 0 \leqslant L \leqslant 2 .
$$

We shall find out for which $L$ this particular sequence $g^{(n)}$ is a sequence of right tails for a convergent continued fraction (0.1). Since

$$
a_{n}=g^{(n-1)}\left(1+g^{(n)}\right)=-\frac{1}{4}-\frac{(2-L) L}{16 n(n+1)}, \quad n \geqslant 1,
$$

we know by [12] (see also [14]) that the continued fraction $K\left(a_{n} / 1\right)$ converges for all $L \in[0,2]$. From [14] we even know the right tails

$$
f^{(n)}=-\frac{1}{2}-\frac{1-\sqrt{1-(2-L) L}}{4(n+1)}=-\frac{1}{2}-\frac{1-|1-L|}{4(n+1)},
$$

and hence

$$
f^{(n)}= \begin{cases}-\frac{1}{2}-\frac{L}{4(n+1)} & \text { for } 0 \leqslant L \leqslant 1, \\ -\frac{1}{2}-\frac{2-L}{4(n+1)} & \text { for } 1<L \leqslant 2 .\end{cases}
$$

Thus $\left\{g^{(n)}\right\}$ is a sequence of right tails if $0 \leqslant L \leqslant 1$ and of wrong tails if $1<L \leqslant 2$. We shall see that this is what we get by using Theorem 1 . We have in this case for $L \leqslant 1$

$\kappa_{n}=\frac{1}{2}-4(n+1)^{-L} / \frac{1}{2}+4(n+1)^{-L} \geqslant \frac{1}{2}-4(n+1)^{-1} / \frac{1}{2}+4(n+1)^{-1}=\frac{2 n+1}{2 n+3}$, and

$$
\kappa_{1} \kappa_{2} \cdots \kappa_{n} \geqslant \frac{3}{2 n+3} .
$$

Hence the $\kappa$-series converges to $\infty$, and the tails are right. If we replace $L$ by $2-L$, we find for $L>1$ the inequality (3.8). Hence $-\frac{1}{2}-(2-L) / 4(n+1)$ is right for $1<L \leqslant 2$ and thus $-\frac{1}{2}-L / 4(n+1)$ is wrong.

4. Final remarks. The tails are closely related to the parameters of Wall's chain sequences $\left[15\right.$, p. 79], i.e. sequences $\left(1-g_{0}\right) g_{1},\left(1-g_{1}\right) g_{2},\left(1-g_{2}\right) g_{3}, \ldots$, where $0 \leqslant g_{p} \leqslant 1$. The connection is

$$
g_{p}-1=g^{(p)}
$$


and

$$
\left(1-g_{p}\right) g_{p+1}=-g^{(p)}\left(1+g^{(p+1)}\right)=-a_{p+1} .
$$

In discussing the relations between chain sequences and tails we are thus restricted to tails $g^{(n)} \in[-1,0]$ and thus $a_{n} \in\left[-\frac{1}{4}, 0\right]$. This, and also relations to results by Chihara [1-3] on chain sequences will be taken up in a later paper.

The author is indebted to L. Jacobsen for calling my attention to [11, Satz 2.45].

\section{REFERENCES}

1. T. S. Chihara, Chain sequences and orthogonal polynomials, Trans. Amer. Math. Soc. 104 (1962), $1-16$.

2. $362-371$

3. , Orthogonal polynomials whose zeros are dense in intervals, J. Math. Anal. Appl. 24 (1968),

Anal. 11 (1980) 358-364.

4. Lisa Jacobsen, Convergence acceleration for continued fractions $K\left(a_{n} / 1\right)$, Trans. Amer. Math. Soc. 275 (1983), 265-285.

5. $\quad$ A method for convergence acceleration of continued fractions $K\left(a_{n} / 1\right)$, Lectures Notes in Math., 932, Springer-Verlag, Berlin and New York, 1982, pp. 74-86.

6. Further results on convergence acceleration for continued fraction $K\left(a_{n} / 1\right)$, Trans. Amer.

Math. Soc. (to appear).

7. Functions defined by continued fractions. Meromorphic continuation (submitted).

8. Convergence of limit k-periodic continued fractions $K\left(a_{n} / b_{n}\right)$ and of subsequences of their tails (in preparation).

9. Lisa Jacobsen and Haakon Waadeland, Some useful formulas involving tails of continued fractions, Lecture Notes in Math., vol. 932, Springer-Verlag, Berlin and New York, 1982, pp. 99-105.

10. William B. Jones and Wolfgang J. Thron. Continued fractions: analytic theory and applications. Encyclopedia Math. Appl., No. 11, Addison-Wesley, Reading, Mass., 1980.

11. O. Perron, Die Lehre von den Kettenbrüchen, 3. Aufl., 2. Band, Teubuer, Stuttgart, 1957.

12. A. Pringsheim, Über die Konvergenz unendlicher Kettenbrüche, Bayer. Akad. Nat. Wiss. Kl. Sitz. Ber. 28 (1899), 295-324.

13. W. J. Thron and Haakon Waadeland, Modifications of continued fractions, a survey, Lecture Notes in Math., vol. 932, Springer-Verlag, Berlin and New York, 1982, pp. 38-66.

14. On a certain transformation of continued fractions, Lecture Notes in Math., vol. 932. Springer-Verlag, Berlin and New York, 1982, pp. 225-240.

15. H. S. Wall, Analytic theory of continued fractions, Chelsea, New York, 1967.

Department of Mathematics and Statistics, University of Trondheim, N-7055 Dragvoll, NORWAY 